\title{
Effects of Pulsatile Perfusion upon Left Ventricular Function ${ }^{1}$
}

\author{
Jeffrey Dunn, M.D., ${ }^{*}$ Alan Peterson, M.D., $\dagger$ \\ and Marvin M. Kirsh, M.D., F.A.C.S.* \\ The *Department of Surgery, Sections of Thoracic Surgery and $\dagger$ General Surgery, The University of Michigan \\ Medical Conter, Ann Arbor, Michigan 48109
}

Submitted for publication August 16, 1977

\begin{abstract}
This study compares the effects of pulsatile and nonpulsatile perfusion upon myocardial contractility. After $2 \mathrm{hr}$ of ventricular fibrillation, control animals demonstrated a $37 \%$ decrease in left ventricular force, a $48 \%$ decrease in left ventricular compliance, and an increase in endomyocardial water content. On the other hand, after $2 \mathrm{hr}$ of ventricular fibrillation, animals undergoing pulsatile perfusion had no significant change in ventricular diastolic compliance and only a $14 \%$ decrease in left ventricular force. The results of this study show that the addition of pulsatile perfusion minimized the deleterious effects of ventricular fibrillation upon myocardial contractility.
\end{abstract}

\section{INTRODUCTION}

The ideal method for myocardial protection during extracorporeal circulation has not yet been established. The detrimental effects of prolonged ventricular fibrillation upon ventricular function during extracorporeal circulation are well recognized $[4,5]$. However, at times it is impossible to avoid ventricular fibrillation during a cardiac operation.

One can easily convert nonpulsatile cardiopulmonary bypass to pulsatile flow by the incorporation of a pulsatile ventricle activated by an intra-aortic balloon pump in the arterial perfusion line, even in the presence of ventricular fibrillation [3].

The purpose of this experiment was to compare the effects of pulsatile perfusion on myocardial contractility during ventricular fibrillation against the effects of nonpulsatile perfusion during $2 \mathrm{hr}$ of normothermic cardiopulmonary bypass.

\section{METHODS}

Twelve adult mongrel dogs weighing between 25 and $35 \mathrm{~kg}$ were anesthetized with

${ }^{1}$ Reprint requests should be addressed to Marvin M. Kirsh, M.D., C7163 Out-Paticnt Building, Section of Thoracic Surgery, University Hospital, Ann Arbor, Mich. 48109.
Dialurethane $(0.6 \mathrm{ml} / \mathrm{kg}$ body $\mathrm{wt})$ and mechanically ventilated with a mixture of $70 \%$ nitrous oxide and $30 \%$ oxygen. Polyvinyl catheters were placed in the femoral vein and carotid artery for measurement of arterial and venous systemic pressure. A median sternotomy was performed, the azygos vein was ligated, and polyethylene tapes were placed around the superior and inferior vena cava and aorta.

A pump oxygenator system ${ }^{2}$ was used to establish cardiopulmonary bypass. The oxygenator was primed with $2000 \mathrm{cc}$ of Ringer's lactate solution. After the animal was heparinized, the superior and inferior venae cavae were cannulated through the right atrium, and an arterial perfusion cannula was placed in the ascending aorta. The sinoatrial node was crushed and a steady heart rate was maintained with the use of atrial pacing at a rate of $160 \pm 20$ beats per minute. The animals were then placed on partial cardiopulmonary bypass, maintaining the systemic perfusion pressure initially at $80 \mathrm{~mm} \mathrm{Hg}$ and the temperature at $37^{\circ} \mathrm{C}$ with a heat exchanger. A left ventricular sump catheter was inserted through the apex of the left ventricle in order to decompress it.

\footnotetext{
${ }^{2}$ Harvey bubble oxygenator and Sarns roller pump model 5000.
} 
The animals were then placed on total cardiopulmonary bypass.

A Walton-Brody strain gauge was sutured in place on the surface of the left ventricle just distal to the first diagonal branch of the left anterior descending coronary artery. A 40-cc latex balloon catheter was inserted through the apex of the heart, adjacent to the sump, for pressure determination. Pressures were measured with Statham transducers and recorded on an Electronics for Medicine recorder. Left ventricular compliance was assessed by inflating the intraventricular balloon $5 \mathrm{cc}$ at a time to $25 \mathrm{cc}$ and recording its corresponding left ventricular enddiastolic pressure. The Walton-Brody strain gauge was calibrated with gram weights and recorded on the Electronics for Medicine recorder. The Walton-Brody strain gauge measurements were also recorded at varying ventricular volumes. The studies were done 15 min after institution of total cardiopulmonary bypass with the heart beating at a perfusion pressure of $80 \mathrm{~mm} \mathrm{Hg}$.

After measurements of these parameters, the heart was electrically fibrillated, the perfusion pressure was reduced to a mean pressure of $50 \mathrm{~mm} \mathrm{Hg}$, and maintained at this level in all 12 animals throughout the period of ventricular fibrillation. The animals were randomly divided into groups.

Group I $(n=6)$ served as the control animals and underwent $2 \mathrm{hr}$ of nonpulsatile perfusion.

Group II $(n=6)$ served as the experimental animals in which pulsatile flow was produced by placing a pulsatile ventricle in series in the arterial perfusion line. The ventricle was activated by an Avco intraaortic balloon pump at a fixed rate of 100 beats $/ \mathrm{min}$. The pulsatile flow had a pulse amplitude of $40 \mathrm{~mm} \mathrm{Hg}$ with the mean pressure being maintained at $50 \mathrm{~mm} \mathrm{Hg}$.

After $2 \mathrm{hr}$ of ventricular fibrillation, the animals in both groups were electrically defibrillated and maintained at a mean perfusion pressure of $80 \mathrm{~mm} \mathrm{Hg}$ and rested for $15 \mathrm{~min}$. The previously described measurements were repeated.
The animals were then killed and a portion of the free left ventricular wall between the anterior and posterior papillary muscles was removed, divided into epicardial and endocardial portions, and weighed immediately. The samples were dried at $45^{\circ} \mathrm{C}$ to a constant weight. Percent of water content was then determined. All experimental determinations were tested by Student's $t$ test. All statistical determinations of $p<0.05$ are considered significant; those of $p<0.1$ are considered to approach significance.

\section{RESULTS}

Prior to ventricular fibrillation there was no difference in left ventricular force between the two groups (Fig. 1). After $2 \mathrm{hr}$ of ventricular fibrillation, the left ventricular force decreased an average of $37 \%$ in Group I animals.

This decrease in left ventricular force in Group I animals occurred at all end-diastolic volumes. The most striking change occurred at an end-diastolic volume of $15 \mathrm{cc}$, when the left ventricular force fell from $171.9 \pm 2.40$ to $104.7 \pm 18 \mathrm{~g}$, which represented a $61 \%$ decrease $(p<0.05)$.

The $d F / d t$ value is obtained in grams per second by electronically differentiating the Walton-Brody force curve (Fig. 2). Prior to ventricular fibrillation, there was no difference in $d F / d t$ between the two groups. After $2 \mathrm{hr}$ of ventricular fibrillation, $d F / d t$ in Group I animals was reduced by $39.6 \%(p<0.05)$. This decrease in $d F / d t$ occurred at all enddiastolic volumes. At an end-diastolic volume of $15 \mathrm{cc}$, the $d F / d t$ fell from 3912 \pm 451 to $1797 \pm 340 \mathrm{~g} / \mathrm{sec}$, which represented a $45.9 \%$ decrease.

Conversely, this deleterious effect on ventricular function was markedly lessened in the fibrillating hearts with pulsatile perfusion (Figs. 1 and 2). In Group II animals the left ventricular force averaged $194.7 \mathrm{~g}$ prior to ventricular fibrillation and $162.6 \mathrm{~g}$ after $2 \mathrm{hr}$ of ventricular fibrillation with pulsatile perfusion (N.S.). At all end- 


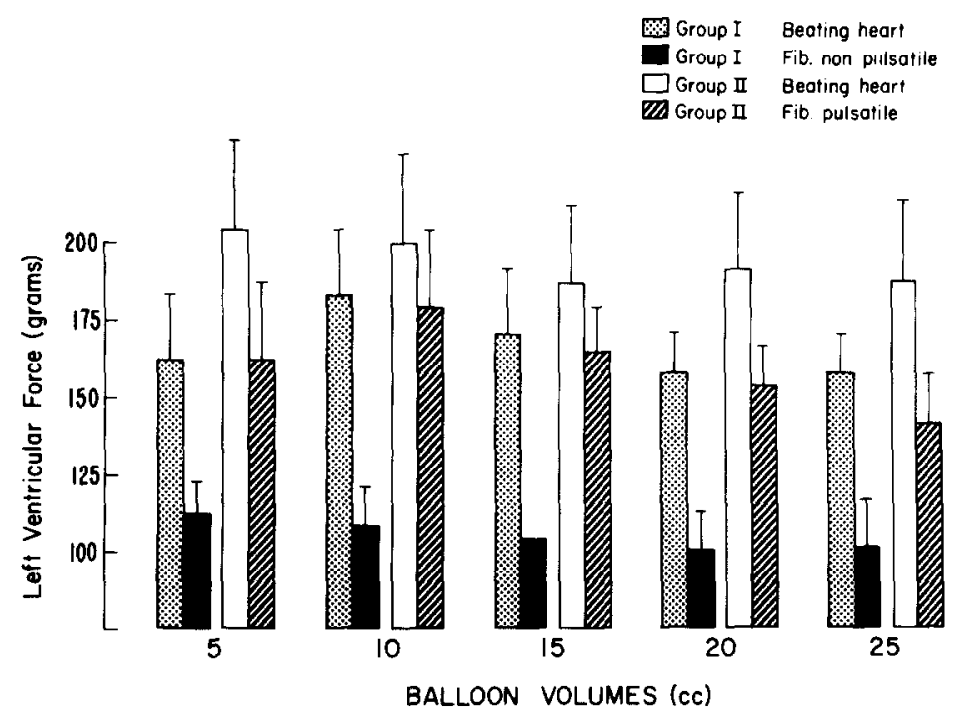

FIG. 1. The effect of pulsatile perfusion during ventricular fibrillation upon left ventricular force. Note that left ventricular force did not decrease significantly in animals undergoing pulsatile perfusion.

diastolic volumes there was no statistically significant decrease in left ventricular force.

Similarly, the $d F / d t$ was preserved in the animals undergoing pulsatile perfusion. Prior to ventricular fibrillation, $d F / d t$ averaged 2776 and $2468 \mathrm{~g} / \mathrm{sec}$ after $2 \mathrm{hr}$ of pulsatile perfusion. Also, at all end-diastolic volumes, there was no statistically significant decrease in $d F / d t$ (Fig. 2).
Left ventricular compliance was observed in the two groups. In Group I, ventricular diastolic compliance decreased an average of $48.9 \%$ when the postperfusion values were compared to the preventricular fibrillation values. The left ventricular compliance of Group II animals in essence remained unchanged with time (Table 1).

There was no difference between the two

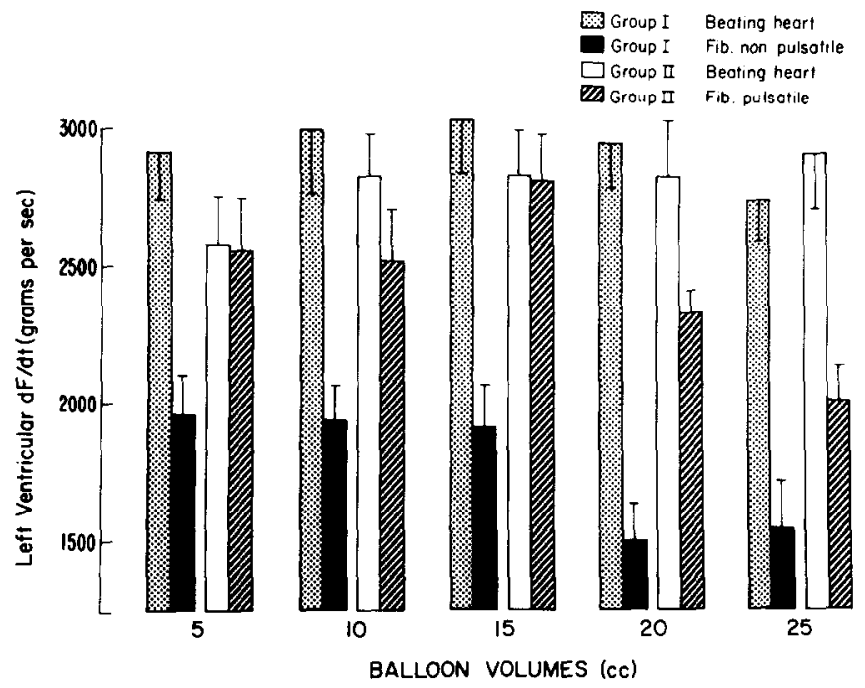

FIG. 2. The effect of pulsatile perfusion during ventricular fibrillation upon $d F / d t$. Note that $d F / d t$ did not decrease significantly in animals undergoing pulsatile perfusion. 
TABLE 1

Left Ventricular End-Diastolic Pressure (mm Hg)

\begin{tabular}{crrrrr}
\hline \multirow{2}{*}{$\begin{array}{c}\text { Diastolic } \\
\text { volume } \\
(\mathrm{cc})\end{array}$} & \multicolumn{2}{c}{ Group I } & & \multicolumn{2}{c}{ Group II } \\
\cline { 2 - 3 } \cline { 5 - 6 } & Pre-vent fib & Postbypass & & Pre-vent fib & Postbypass \\
\hline 5 & $0.7 \pm 2.1$ & $0.2 \pm 1.6$ & & $3.0 \pm 1.7$ & $4.4 \pm 1.6$ \\
10 & $3.5 \pm 1.0$ & $10.4 \pm 3.3$ & & $7.0 \pm 1.1$ & $8.3 \pm 1.0$ \\
15 & $7.0 \pm 0.9$ & $12.4 \pm 3.2$ & & $9.3 \pm 1.1$ & $10.7 \pm 0.8$ \\
20 & $11.2 \pm 3.0$ & $20.0 \pm 6.1$ & & $8.7 \pm 1.1$ & $11.7 \pm 1.3$ \\
25 & $15.8 \pm 5.6$ & $26.4 \pm 9.2$ & & $11.0 \pm 1.2$ & $15.5 \pm 2.5$ \\
\hline
\end{tabular}

groups postperfusion with reference to either left ventricular systolic pressure or left ventricular $d p / d t$ (Figs. 3 and 4).

\section{Myocardial Water Content}

The endomyocardial water content following nonpulsatile perfusion averaged 78.45 \pm 0.39 and $76.40 \pm 37$ in the animals undergoing pulsatile perfusion $(p<0.01)$. However, there was no statistically significant difference in epicardial water content between the two groups $(76.18 \pm .03$ vs $75.24 \pm 0.90$ ).

\section{DISCUSSION}

Studies by Hottenrott and Habal and others have demonstrated the detrimental effects of ventricular fibrillation on distribution of coronary blood flow, myocardial metabolism, and ventricular function in experimental animals [3-5]. Hottenrott found that $1 \mathrm{hr}$ of continuous ventricular fibrillation resulted in a fall in myocardial oxygen consumption and depression in left ventricular function as compared to that of the empty, beating, working heart. These adverse effects were exaggerated by preexisting myocardial hypertrophy [4]. Habal reported that $120 \mathrm{~min}$ of ventricular fibrillation resulted in a decrease in ventricular diastolic compliance, an increase in oxygen consumption, decrease in lactate extraction, and a decrease in proportions of total left ventricular flow delivered to the subendocardium as compared to that of the empty, beating, working heart [3].
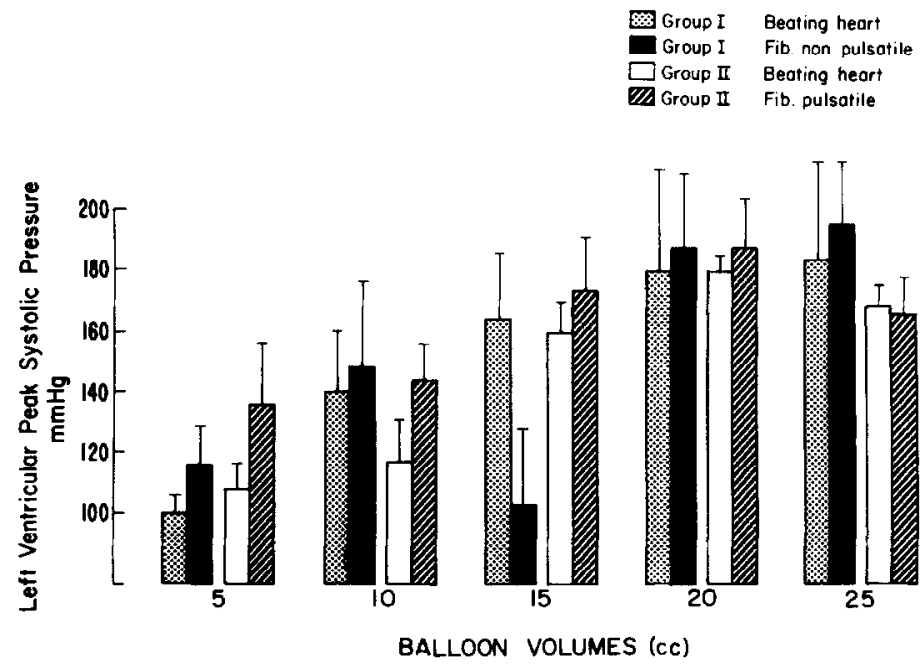

BALLOON VOLUMES (cc)

FIG. 3. The effect of pulsatile perfusion during ventricular fibrillation upon left ventricular peak systolic pressure. 

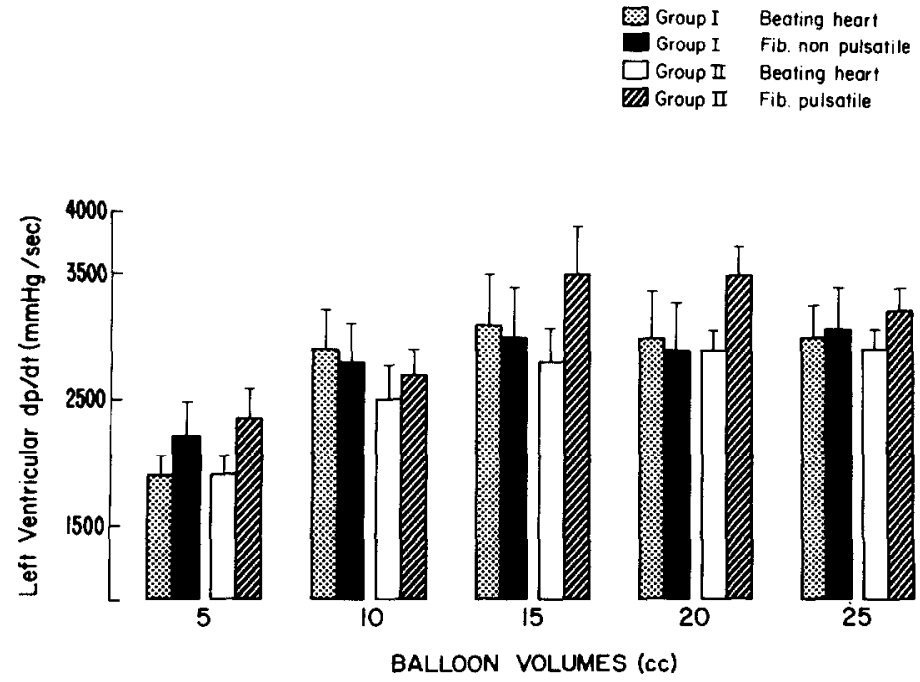

FIG. 4. The effect of pulsatile perfusion during ventricular fibrillation upon left ventricular $d p / d t$.

The advantages of pulsatile over nonpulsatile perfusion have been previously described and include better capillary perfusion, improved tissue and acid-base status, increased oxygen consumption, lower peripheral vascular resistance, lower transfusion volume, higher flow rates, and improved cerebral and renal perfusion [1]. Despite the fact that there have been numerous publications concerning the value of pulsatile perfusion during cardiopulmonary bypass, there have been relatively few studies concerning the effects of pulsatile perfusion on myocardial function as such. Pappas and associates demonstrated in man that intra-aortic balloon pumping during cardiopulmonary bypass converts nonpulsatile flow to pulsatile flow [7]. Ventricular fibrillation was used in these patients and the aorta was not clamped. These authors found that pulsatile flow resulted in less elevation of coronary sinus lactate and a lower incidence of peri-operative myocardial infarction during cardiopulmonary bypass when compared to nonpulsatile perfusion in a similar group of patients. Maddoux, using a radionuclide technique, measured left ventricular ejection fraction in $\mathbf{4 0}$ patients undergoing myocardial revascularization [6]. In the group of patients receiving pulsatile flow, there was an improvement of ejection fraction postoperatively, whereas in the group experiencing nonpulsatile flow, the ejection fraction decreased.

Habal and associates compared the effects of pulsatile and nonpulsatile flow on myocardial performance during $2 \mathrm{hr}$ of normothermic ventricular fibrillation in animals [3]. Pulsatile flow was delivered through a modified Bentley pulsatile pump, pneumatically activating a pulsatile ventricle incorporated into the coronary perfusion line. These authors found that the addition of pulsatile flow to coronary perfusion during ventricular fibrillation resulted in normal left ventricular compliance, improved myocardial lactate and oxygen extraction, and reduced ischemic subendocardial histologic changes.

Similarly, the present experiment demonstrates that the detrimental effects of $2 \mathrm{hr}$ of ventricular fibrillation on myocardial function were minimized by the use of pulsatile perfusion. The pulsatile perfusion group has less myocardial edema than the nonpulsatile group and, consequently, a better preservation of ventricular diastolic compliance. The decrease in compliance and increase in myocardial water content that occurred in the group undergoing nonpulsatile perfusion reflects the occurrence of myocardial ischemia 
during perfusion. Pulsatile perfusion was also associated with preservation of myocardial contractility. Left ventricular force decreased $37 \%$ in the animals perfused with nonpulsatile flow, whereas left ventricular force decreased only $14 \%(p<.05)$ postbypass in the animals undergoing pulsatile perfusion. Likewise the rate of force production $(d F / d t)$ was better preserved in the pulsatile perfusion group.

Until recently, available pulsatile pumps were not satisfactory for clinical use because they were associated with excessive hemolysis, low output capacity, hazards of air from hydraulic embolus, or mechanical complexity. With the development of pulsatile assist devices or the addition of intraaortic balloon pumping to the bypass system, pulsatile flow can now be produced during cardiopulmonary bypass with systems that are reliable, safe, and as simple to regulatc as the standard roller pumps that are in use today. Because of the advantages of pulsatile flow in preserving myocardial function during ventricular fibrillation demonstrated in this and other studies, it should be the method of choice for patients undergoing open heart surgery in whom ventricular fibrillation will be utilized.

\section{REFERENCES}

1. Dunn, J., Kirsh, M., Harness, J., Carrol, M., Straker, J., and Sloan, H. Hemodynamic, metabolic, and hematologic effects of pulsatile cardiopulmonary bypass. J. Thorac. Cardiovasc. Surg. 68: $138,1974$.

2. Habal, S., Weiss, M., Spotnetz, H., Parodi, E., Wolff, M., Canna, P., Hoffman, B., and Malm, J. Effects of pulsatile and nonpulsatile coronary perfusion on the performance of the left ventricle. J. Thor. Cardiovasc. Surg. 72: 742, 1976.

3. Habal, S., Parodi, E., Hansen, B., Wolvek, S., and Bregman, D. A new pulsatile assert device (PAD) for use in cardiac surgery. 22nd Annual Meeting, Amer. Soc. Artif. Internal Organs, 1976.

4. Hottenrott, R., Towers, B., Kurkji, H., Maloney, J., and Buckberg, G. The hazards of ventricular fibrillation in hypertrophied ventricles during cardiopulmonary bypass. J. Thorac. Cardiovasc. Surg. 66: 742, 1973.

5. Hottenrott, D., and Buckberg, G. Studies of the effects of ventricular fibrillation on the adequacy of regional myocardial flow. J. Thorac. Cardiovasc. Surg. 68: 626, 1974.

6. Maddoux, G., Pappas, G., and Jenkins, M. Comparison of pulsatile and nonpulsatile flow during cardiopulmonary bypass on left ventricular ejection fraction early after aortocoronary surgery. Amer. J. Card. 37: 1000, 1976.

7. Pappas, G., Winter, S., Kopriva, C., and Steele, P. Improvement of myocardial and other vital organ functions and metabolism with a sample method of pulmonary flow (IABP) during clinical cardiopulmonary bypass. Surgery 77: 34, 1975. 